\title{
Configuration Mixing of Quark States in Baryons ${ }^{\star}$
}

\author{
B. Saghai Saghai
}

Institut de Recherche sur les lois

Fondamentales de l'Univers,

DSM/Irfu, CEA/Saclay, 91191 Gif-sur-Yvette,

France

E-mail: bijan.saghai@cea.fr

and

Z. Li

SGT Inc, 7701 Greenbelt Road, Greenbelt, MD. 20770, USA

the date of receipt and acceptance should be inserted later

\begin{abstract}
Mixing angles are used to describe the $S U(6) \otimes O(3)$ symmetry breaking in $\left[70,1^{-}\right]$multiplet in the sector of the lowest mass nucleon resonances, which are investigated extensively in constituent quark models for baryon spectroscopy. The transition amplitudes for the meson photoproduction off nucleon can also be expressed in terms of the mixing angles to take into account the configuration mixing. Those amplitudes are derived as a function of the mixing angles between $\left|N^{2} P_{M} J^{-}\right\rangle$and $\left|N^{4} P_{M} J^{-}\right\rangle$ states, with $J=1 / 2$ and $3 / 2$, for the processes $\gamma p \rightarrow \eta p, K^{+} \Lambda, K^{+} \Sigma^{\circ}, K^{\circ} \Sigma^{+}$. The present status of our knowledge on the mixing angles between $S_{11}(1535)$ and $S_{11}(1650)$ $\left(\theta_{S}\right)$, as well as between $D_{13}(1520)$ and $D_{13}(1700)\left(\theta_{D}\right)$ is reported. Since these resonances play very important role in the threshold region for both $\eta$ and kaon production mechanisms, they are expected to provide crucial tests of different quark models for the baryon spectroscopy.
\end{abstract}

Keywords Baryon resonances · chiral quark models · Symmetry breaking

PACS 14.20.Gk $\cdot$ 12.39.Fe $\cdot$ 11.30.Qc

\section{Introduction}

Discovery of the first baryon resonance, $\Delta$, goes back to 1952 , when Fermi and collaborators released [1] unexpected experimental results on the $\pi^{ \pm} p$ interactions, followed by Impulse approximation [2] and phase shift analysis [3,4,5]. In the late 1950s and early 1960s the road to The Eightfold way was paved and by the mid-sixties a proliferation of discovery of mesons and baryons, including nucleon resonances, received a

* Manuscript dedicated to Professor Willibald Plessas for his sixtieth birthday. Published in: Few-Body Syst 47, 105 (2010).

Address(es) of author(s) should be given 
genuine classification, based on the $\mathrm{SU}(3)$ symmetry and the concept of quarks [6.7], as the elementary blocks of hadrons.

A natural extension of the $\mathrm{SU}(3)$ flavor symmetry, taking into account the fermion nature of quarks, lead then to the product subgroup $S U(3) \otimes S U(2)$, and hence to an $S U(6)$ flavor-spin symmetry structure of the strongly interacting particles 8 . Finally, the intrinsic spin group $S U(2)$ and the internal symmetry group $S U(3)$, were complemented with a group of rotations in the three-dimensional space $O(3)$ symmetry, suggesting the invariance of strong interactions under the $S U(6) \otimes O(3)$ symmetry 9 .

The early works by Copley et al. [10] and Feynman et al. 11] on the pion photoproduction, provided the first clear evidence of the underlying $S U(6) \otimes O(3)$ structure of the baryon spectrum.

Those classifications initiated dynamical approaches with regards to the baryon spectroscopy, the outcomes of which go beyond the known resonances [12, predicting still undiscovered or "missing" resonances.

Here, we concentrate on the features of the baryon spectroscopy arising from the configuration mixings. For the $S$ - and $D$-wave resonances classified as $\left[70,1^{-}\right]$multiplet, the configuration mixings can be expressed as the mixing angles, which have been predicted by various quark models based on the baryon mass spectrum.

In this paper, we present a frame to investigate the configuration mixings in meson photoproduction within a constituent quark model and generated by the $S U(6) \otimes$ $O(3)$ symmetry breaking. The transition amplitudes for the resonances belonging to $\left[70,1^{-}\right]$multiplet are expressed in terms of the mixing angles, which can be extracted from meson photoproduction. Comparing to the studies [13, 14, 15, 16, 17, 18, 19, 20, 21 . on the baryon spectroscopy and transition amplitudes that are static, meson photoproduction processes offer additional tests on the mixing angles. In particular, the close to threshold behavior of $\eta$ 22, 23, 24, 25, 26, 27, 28, 29, 30,31] and, to a lesser extent, of kaon photoproduction $32,33,34$ reactions are largely dominated by the $S$ - and $D$-wave resonances, which are very sensitive to the values and signs of the mixing angles. For the latter reaction, a "selection rule" has recently been suggested 35. for $N^{*}$ resonances in the presence of QCD mixing effects.

The paper is organized as follows. In Section 2 first the expressions for configuration mixing and the related angles are recalled (Sec. 2.1), then we briefly present a chiral constituent quark model, introduce a $S U(6) \otimes O(3)$ symmetry breaking coefficient, and relate it to the configuration mixing angles via a constant $\mathcal{R}$ (Sec. 2.2). The main novelty of the present work is reported in Sec. 2.3. where we derive explicit expressions for the constant $\mathcal{R}$, establishing relations among the $S U(6) \otimes O(3)$ symmetry breaking coefficients and the mixing angles, within pseudoscalar mesons photoproduction processes. Section 3 is devoted to the determination of the mixing angles, and conclusions are given in Sec. 4 .

\section{Configuration Mixing}

For the $S U(6) \otimes O(3)$ states, we use the general notation $X\left({ }^{2 S+1} L_{\pi}\right)_{J^{P}}$, with $X \equiv N$ or $\Delta, S$ the quark spin, $L=S, P, D, \ldots$ the orbital angular momentum, $\pi \equiv S, M, A$ the permutation symmetry (symmetric, mixed, antisymmetric) of the spatial wave function, and $J^{P}$ the state's total angular momentum and parity. The wave functions for isospin-1/2 resonances with masses below $2 \mathrm{GeV}$ are given in Table 1 
Here, the most relevant configuration mixings are those of the two $S_{11}$ and the two $D_{13}$ states around 1.5 to $1.7 \mathrm{GeV}$ (Table1). The configuration mixings can be expressed in terms of the mixing angle between the two $S U(6) \otimes O(3)$ states $\mid N\left({ }^{2} P_{M}\right)_{J^{P}}>$ and $\mid N\left({ }^{4} P_{M}\right)_{J^{P}}>$, with the total quark spin $J=1 / 2$ and $3 / 2$;

$$
\left(\begin{array}{l}
\mid S_{11}(1535)> \\
\mid S_{11}(1650)>
\end{array}\right)=\left(\begin{array}{cc}
\cos \theta_{S} & -\sin \theta_{S} \\
\sin \theta_{S} & \cos \theta_{S}
\end{array}\right)\left(\begin{array}{l}
\mid N\left({ }^{2} P_{M}\right)_{\frac{1}{2}}{ }^{-}> \\
\mid N\left({ }^{4} P_{M}\right)_{\frac{1^{-}}{}}{ }^{-}>
\end{array}\right),
$$

and

$$
\left(\begin{array}{l}
\mid D_{13}(1520)> \\
\mid D_{13}(1700)>
\end{array}\right)=\left(\begin{array}{cc}
\cos \theta_{D} & -\sin \theta_{D} \\
\sin \theta_{D} & \cos \theta_{D}
\end{array}\right)\left(\begin{array}{l}
\mid N\left({ }^{2} P_{M}\right)_{\frac{3}{2}}-> \\
\mid N\left({ }^{4} P_{M}\right)_{\frac{3}{2}}{ }^{-}>
\end{array}\right),
$$

where $\theta_{S}$ and $\theta_{D}$ are the mixing angles between the $S_{11}$ resonances and the $D_{13}$ resonances, respectively.

Table 1 Nucleon resonances with their assignments in $S U(6) \otimes O(3)$ configurations.

\begin{tabular}{llllll}
\hline State & $S_{11}(1535)$ & $S_{11}(1650)$ & $D_{13}(1520)$ & $D_{13}(1700)$ & $D_{15}(1675)$ \\
$S U(6) \otimes O(3)$ & $N\left({ }^{2} P_{M}\right)_{\frac{1}{2}}{ }^{-}$ & $N\left({ }^{4} P_{M}\right)_{\frac{1}{2}}-$ & $N\left({ }^{2} P_{M}\right)_{\frac{3}{2}}-$ & $N\left({ }^{4} P_{M}\right)_{\frac{3}{2}}-$ & $N\left({ }^{4} P_{M}\right)_{\frac{5}{2}}{ }^{-}$ \\
State & $P_{13}(1720)$ & $F_{15}(1680)$ & $P_{11}(1440)$ & $P_{11}(1710)$ & \\
$S U(6) \otimes O(3)$ & $N\left({ }^{2} D_{S}\right)_{\frac{3}{2}}{ }^{+}$ & $N\left({ }^{2} D_{S}\right)_{\frac{5}{2}}{ }^{+}$ & $N\left(S_{S}^{\prime}\right)_{\frac{1}{2}}{ }^{+}$ & $N\left({ }^{2} S_{M}\right)_{\frac{1}{2}}{ }^{+}$ & \\
State & $P_{13}(1900)$ & $F_{15}(2000)$ & & & \\
$S U(6) \otimes O(3)$ & $N\left(^{2} D_{M}\right)_{\frac{3}{2}}{ }^{+}$ & $N\left(^{2} D_{M}\right)_{\frac{5}{2}}{ }^{+}$ & & & \\
\hline
\end{tabular}

\subsection{Chiral constituent quark approach}

In this Section, we investigate the manifestations of the $S U(6) \otimes O(3)$ symmetry breaking in the $\eta$ and kaon photoproduction processes, where contributions from $S$ - and $D$-wave resonances are very important in the threshold region. First we recall the main content of the theoretical approach used here, a chiral constituent quark model [36], based on an effective chiral Lagrangian 37,

$$
\mathcal{L}=\bar{\psi}\left[\gamma_{\mu}\left(i \partial^{\mu}+V^{\mu}+\gamma_{5} A^{\mu}\right)-m\right] \psi+\cdots,
$$

where vector $\left(V^{\mu}\right)$ and axial $\left(A^{\mu}\right)$ currents read,

$$
V^{\mu}=\frac{1}{2}\left(\xi \partial^{\mu} \xi^{\dagger}+\xi^{\dagger} \partial^{\mu} \xi\right), \quad A^{\mu}=\frac{1}{2 i}\left(\xi \partial^{\mu} \xi^{\dagger}-\xi^{\dagger} \partial^{\mu} \xi\right),
$$

with $\xi=\exp \left(i \Pi / f_{m}\right)$ and $f_{m}$ the meson decay constant. $\psi$ and $\phi_{m}$ are the quark and meson fields, respectively The field $\Pi$ is a $3 \otimes 3$ matrix,

$$
\Pi=\left(\begin{array}{ccc}
\frac{1}{\sqrt{2}} \pi^{\circ}+\frac{1}{\sqrt{6}} \eta & \pi^{+} & K^{+} \\
\pi^{-} & -\frac{1}{\sqrt{2}} \pi^{\circ}+\frac{1}{\sqrt{6}} \eta & K^{\circ} \\
K^{-} & \bar{K}^{\circ} & -\sqrt{\frac{2}{3}} \eta
\end{array}\right),
$$


in which the pseudoscalar mesons, $\pi, \eta$, and $K$, are treated as Goldstone bosons so that the Lagrangian in Eq. (3) is invariant under the chiral transformation. Therefore, there are four components for the photoproduction of pseudoscalar mesons based on the QCD Lagrangian,

$$
\begin{aligned}
\mathcal{M}_{f i}= & \left\langle N_{f}\left|H_{m, e}\right| N_{i}\right\rangle+\sum_{j}\left\{\frac{\left\langle N_{f}\left|H_{m}\right| N_{j}\right\rangle\left\langle N_{j}\left|H_{e}\right| N_{i}\right\rangle}{E_{i}+\omega-E_{j}}+\right. \\
& \left.\frac{\left\langle N_{f}\left|H_{e}\right| N_{j}\right\rangle\left\langle N_{j}\left|H_{m}\right| N_{i}\right\rangle}{E_{i}-\omega_{m}-E_{j}}\right\}+\mathcal{M}_{T},
\end{aligned}
$$

where $N_{i}\left(N_{f}\right)$ is the initial (final) state of the nucleon, and $\omega\left(\omega_{m}\right)$ represents the energy of incoming (outgoing) photons (mesons). The pseudovector and electromagnetic couplings at the tree level are given, respectively, by the following standard expressions:

$$
H_{m}=\sum_{j} \frac{1}{f_{m}} \bar{\psi}_{j} \gamma_{\mu}^{j} \gamma_{5}^{j} \psi_{j} \partial^{\mu} \phi_{m}, H_{e}=-\sum_{j} e_{j} \gamma_{\mu}^{j} A^{\mu}(\mathbf{k}, \mathbf{r}),
$$

with $j$ the constituent quark index, $\mu$ the space-time coordinate index, and $A^{\mu}(\mathbf{k}, \mathbf{r})$ the electromagnetic field. The first term in Eq. (6) is a seagull term. The second and third terms correspond to the $s$ - and $u$-channels, respectively. The last term is the $t$-channel contribution.

In this paper we focus on the nucleon resonance contributions. For $s$-channel, the transition amplitudes for a resonance are given by the following expression 22,36 :

$$
\mathcal{M}_{N^{*}}=\frac{2 M_{N^{*}}}{s-M_{N^{*}}^{2}-i M_{N^{*}} \Gamma(\mathbf{q})} e^{-\frac{\mathbf{k}^{2}+\mathbf{q}^{2}}{6 \alpha^{2}}} \mathcal{O}_{N^{*}},
$$

where $\sqrt{s} \equiv W=E_{N}+\omega_{\gamma}=E_{f}+\omega_{m}$ is the total centre-of-mass energy of the system, $M_{N^{*}}$ the mass of the resonance, and $\mathcal{O}_{N^{*}}$ is determined by the structure of each resonance and expressed as CGLN amplitudes. The quantity $\mathcal{O}_{N^{*}}$ is also dependent on the photoproduction processes, and explicit expressions for $\mathcal{O}_{N^{*}}$ can be found in Ref. 36 in the $S U(6) \otimes O(3)$ symmetry limit for $\pi, \eta$, and kaon photoproduction. The $\Gamma(\mathbf{q})$ in Eq. (8) is the total width of the resonance, and a function of the final state momentum q. For a resonance decaying into a two-body final state with relative angular momentum $l$, the decay width $\Gamma(\mathbf{q})$ is

$$
\Gamma(\mathbf{q})=\Gamma_{N^{*}} \frac{\sqrt{s}}{M_{N^{*}}} \sum_{i} x_{i}\left(\frac{\left|\mathbf{q}_{i}\right|}{\left|\mathbf{q}_{i}^{N^{*}}\right|}\right)^{2 l+1} \frac{D_{l}\left(\mathbf{q}_{i}\right)}{D_{l}\left(\mathbf{q}_{i}^{N^{*}}\right)},
$$

with

$$
\left|\mathbf{q}_{i}^{N^{*}}\right|=\sqrt{\frac{\left(M_{N^{*}}^{2}-M_{B}^{2}+m_{i}^{2}\right)^{2}}{4 M_{N^{*}}^{2}}-m_{i}^{2}},
$$

and

$$
\left|\mathbf{q}_{i}\right|=\sqrt{\frac{\left(s-M_{B}^{2}+m_{i}^{2}\right)^{2}}{4 s}-m_{i}^{2}},
$$

where $x_{i}$ is the branching ratio of the resonance decaying into a meson with mass $m_{i}$ and a baryon $\left(M_{B}\right)$, and $\Gamma_{N^{*}}$ is the total decay width of the $s$-channel resonance with 
the mass $M_{N^{*}}$. The fission barrier function $D_{l}(\mathbf{q})$ in Eq. (9) is wavefunction dependent. Here we use

$$
D_{l}(\mathbf{q})=e^{-\frac{\mathbf{q}^{2}}{3 \alpha^{2}}}
$$

which is independent of $l$.

Finally, in order to introduce the $S U(6) \otimes O(3)$ symmetry breaking, the transition amplitude $\mathcal{O}_{N^{*}}$ is replaced by the following substitution relation 38]:

$$
\mathcal{O}_{N^{*}} \rightarrow C_{N^{*}} \mathcal{O}_{N^{*}}
$$

where coefficients $C_{N^{*}}$ measure the discrepancies between the theoretical results and the experimental data and show the extent to which the $S U(6) \otimes O(3)$ symmetry is broken in the photon induced processes. In the $S U(6) \otimes O(3)$ symmetry limit, $C_{N^{*}}=0$ for $S_{11}(1650), D_{13}(1700)$, and $D_{15}(1675)$ resonances, and $\left|C_{N^{*}}\right|=1$ for the other resonances, in the mass range $\approx 1.5-2.0 \mathrm{GeV}$, given in Table 1 In the following Section we derive expressions relating the $C_{N^{*}}$ coefficients to the mixing angles.

2.2 Mixing angles and pseudoscalar mesons photoproduction

The scattering amplitudes $\mathcal{O}_{N^{*}}$ expressed in terms of the product of the photon and meson transition amplitudes are

$$
\mathcal{O}_{N^{*}} \propto<N\left|H_{m}\right| N^{*}><N^{*}\left|H_{e}\right| N>
$$

with $H_{m}$ and $H_{e}$ the meson and photon transition operators, respectively. Using the above equations, one finds for the resonance $S_{11}(1535)$

$$
\begin{array}{r}
\mathcal{O}_{S_{11}} \propto<N \mid H_{m}\left[\cos \theta_{S}\left|N\left({ }^{2} P_{M}\right)_{\frac{1}{2}^{-}}>-\sin \theta_{S}\right| N\left({ }^{4} P_{M}\right)_{\frac{1}{2}}->\right] \\
{\left[\cos \theta_{S}<N\left({ }^{2} P_{M}\right)_{\frac{1}{2}}-\left|-\sin \theta_{S}<N\left({ }^{4} P_{M}\right)_{\frac{1}{2}^{-}}\right|\right] H_{e} \mid N>,}
\end{array}
$$

In this approach, the photon transition amplitude $<N\left({ }^{4} P_{M}\right)_{\frac{1}{2}}-\left|H_{e}\right| N>$ vanishes 36] due to the Moorhouse selection rule 39, So, Eq. (15) becomes

$$
\begin{aligned}
\mathcal{O}_{S_{11}} \propto & \left(\cos ^{2} \theta_{S}-\mathcal{R} \sin \theta_{S} \cos \theta_{S}\right)<N\left|H_{m}\right| N\left({ }^{2} P_{M}\right)_{\frac{1}{2}}-> \\
& <N\left({ }^{2} P_{M}\right)_{\frac{1}{2}}-\left|H_{e}\right| N>
\end{aligned}
$$

where $<N\left|H_{m}\right| N\left({ }^{2} P_{M}\right)_{\frac{1}{2}}{ }^{-}><N\left({ }^{2} P_{M}\right)_{\frac{1}{2}}-\left|H_{e}\right| N>$ determines 36] the CGLN amplitude for the $\mid N\left({ }^{2} P_{M}\right)_{\frac{1}{2}^{-}}>$state, and the ratio

$$
\mathcal{R}=\frac{<N\left|H_{m}\right| N\left({ }^{4} P_{M}\right)_{\frac{1}{2}-}>}{<N\left|H_{m}\right| N\left({ }^{2} P_{M}\right)_{\frac{1}{2}^{-}}>},
$$

is a constant determined by the $S U(6) \otimes O(3)$ symmetry. 
The configuration mixing coefficients can be related to the configuration mixing angles

$$
\begin{aligned}
C_{S_{11}(1535)} & =\cos \theta_{S}\left(\cos \theta_{S}-\mathcal{R}_{\mathcal{S}} \sin \theta_{\mathcal{S}}\right), \\
C_{S_{11}(1650)} & =\sin \theta_{S}\left(\mathcal{R}_{\mathcal{S}} \cos \theta_{\mathcal{S}}+\sin \theta_{\mathcal{S}}\right), \\
C_{D_{13}(1520)} & =\cos \theta_{D}\left(\cos \theta_{D}-\mathcal{R}_{\mathcal{D}} \sin \theta_{\mathcal{D}}\right), \\
C_{D_{13}(1700)} & =\sin \theta_{D}\left(\mathcal{R}_{\mathcal{D}} \cos \theta_{\mathcal{D}}+\sin \theta_{\mathcal{D}}\right) .
\end{aligned}
$$

Using the meson transition operator $H_{m}$ from the Lagrangian intervening in deriving the CGLN amplitudes in the quark model, we proceed to the calculation of the $\mathcal{R}$ constant for the $S_{11}$ and $D_{13}$ resonances.

\subsection{Calculation of the $\mathcal{R}$ constant}

The wave functions of the negative-parity $L=1$ nucleon resonances in CQM have the following expressions:

$$
\begin{aligned}
\left|N\left({ }^{4} P_{M}\right)_{J^{-}}\right\rangle= & \frac{1}{\sqrt{2}} \sum_{m}\left\langle J, \frac{1}{2} \mid 1, m, \frac{3}{2}, \frac{1}{2}-m\right\rangle\left(\phi^{\lambda} \psi^{\lambda}+\phi^{\rho} \psi^{\rho}\right) \chi^{S}, \\
\left|N\left({ }^{2} P_{M}\right)_{J^{-}}\right\rangle= & \frac{1}{2} \sum_{m}\left\langle J, \frac{1}{2} \mid 1, m, \frac{1}{2}, \frac{1}{2}-m\right\rangle \\
& {\left.\left[\left(\phi^{\rho} \chi^{\lambda}+\phi^{\lambda} \chi^{\rho}\right) \psi^{\rho}+\left(\phi^{\rho} \chi^{\rho}-\phi^{\lambda} \chi^{\lambda}\right) \psi^{\lambda}\right)\right], }
\end{aligned}
$$

where $\psi, \chi$, and $\phi$ stand for the spatial, spin, and flavor wave functions (Table 2), respectively. Here, $\lambda$ and $\rho$ denote the mixed symmetric and mixed anti-symmetric flavor states, respectively.

Table 2 Flavor wave function for proton and hyperons.

\begin{tabular}{ccc}
\hline State & $\rho$ & $\lambda$ \\
\hline $\mathrm{p}$ & $\frac{1}{\sqrt{2}}($ udu-duu $)$ & $\frac{1}{\sqrt{6}}(2$ uud-duu-udu $)$ \\
$\Lambda$ & $\frac{1}{2 \sqrt{3}}($ usd + sdu-sud-dsu-2dus $+2 \mathrm{uds})$ & $\frac{1}{2}($ sud+usd-sdu-dsu $)$ \\
$\Sigma^{+}$ & $\frac{1}{\sqrt{2}}($ suu-usu $)$ & $\frac{1}{\sqrt{6}}($ suu + usu-2uus $)$ \\
$\Sigma^{\circ}$ & $\frac{1}{2}($ sud + sdu-usd-dsu $)$ & $\frac{1}{2 \sqrt{3}}($ sdu + sud + dsu+usd-2uds-2dus $)$ \\
\hline
\end{tabular}

Moreover

$$
\left|N_{f}\right\rangle=\frac{1}{\sqrt{2}}\left(\phi_{f}^{\lambda} \chi^{\lambda}+\phi_{f}^{\rho} \chi^{\rho}\right) \psi^{S} .
$$

The transition operator can be written as

$$
H_{M} \approx \sum h_{m}^{i} \boldsymbol{\sigma}_{i} \cdot \boldsymbol{p}_{i} e^{i q \cdot r_{i}} \approx h_{m}^{3} \boldsymbol{\sigma}_{3} \cdot \boldsymbol{p}_{3} e^{i q \cdot r_{3}},
$$


with $\boldsymbol{\sigma}_{i}$ spin operator, $\boldsymbol{p}_{i}$ incident beam momentum, $\boldsymbol{q}$ outgoing meson momentum, and $\boldsymbol{r}_{i}$ spatial coordinate. The isospin operators for the pseudoscalar mesons of interest here have the following expressions:

$$
\begin{gathered}
h_{K^{+}}^{3}=a_{3}^{+}(s) a_{3}(u), \\
h_{K^{\circ}}^{3}=a_{3}^{+}(s) a_{3}(d), \\
h_{\eta}^{3}=\left(\left(a_{3}^{+}(u) a_{3}(u)+a_{3}^{+}(d) a_{3}(d)\right) .\right.
\end{gathered}
$$

Here, $a_{3}^{+}(s)$ and $a_{3}(u)\left(a_{3}^{+}(d)\right)$ are the creation and annihilation operators for the strange and up (down) quarks, respectively. So, we have

$$
\begin{aligned}
\left\langle N_{f}\left|H_{k}\right|^{4} N\right\rangle \approx & \frac{1}{2} \sum_{m}\left\langle J, \frac{1}{2} \mid 1, m, \frac{3}{2}, \frac{1}{2}-m\right\rangle\left\langle\phi_{f}^{\lambda}\left|h_{m}^{3}\right| \phi_{N}^{\lambda}\right\rangle\left\langle\chi^{\lambda}\left|\boldsymbol{\sigma}_{3}\right| \chi^{S}\right\rangle \bullet \\
& \left\langle\psi_{f}^{S}\left|\boldsymbol{p}_{3} e^{i q \cdot r_{3}}\right| \psi_{N}^{\lambda}\right\rangle,
\end{aligned}
$$

and

$$
\begin{aligned}
\left\langle N_{f}\left|H_{k}\right|^{2} N\right\rangle \approx & \frac{1}{2 \sqrt{2}} \sum_{m}\left\langle J, \frac{1}{2} \mid 1, m, \frac{3}{2}, \frac{1}{2}-m\right\rangle\left[\left\langle\phi_{f}^{\rho}\left|h_{m}^{3}\right| \phi_{N}^{\rho}\right\rangle\left\langle\chi^{\rho}\left|\boldsymbol{\sigma}_{3}\right| \chi^{\rho}\right\rangle-\right. \\
& \left.\left\langle\phi_{f}^{\lambda}\left|h_{m}^{3}\right| \phi_{N}^{\lambda}\right\rangle\left\langle\chi^{\lambda}\left|\boldsymbol{\sigma}_{3}\right| \chi^{\lambda}\right\rangle\right] \bullet\left\langle\psi_{f}^{S}\left|\boldsymbol{p}_{3} e^{i q . r_{3}}\right| \psi_{N}^{\lambda}\right\rangle
\end{aligned}
$$

The matrix elements $\left\langle\phi_{f}^{\alpha}\left|h_{m}^{3}\right| \phi_{N}^{\alpha}\right\rangle$ are given in Table 3 .

Table 3 Matrix elements $\left\langle\phi_{f}^{\alpha}\left|h_{m}^{3}\right| \phi_{N}^{\alpha}\right\rangle$ for $\eta N$ and kaon-hyperon systems.

\begin{tabular}{ccccc}
\hline State & $\eta N$ & $K^{+} \Lambda$ & $K^{+} \Sigma^{\circ}$ & $K^{\circ} \Sigma^{+}$ \\
\hline$\alpha=\rho$ & 1 & $\sqrt{\frac{2}{3}}$ & 0 & 0 \\
$\alpha=\lambda$ & 1 & 0 & $\frac{\sqrt{2}}{3}$ & $-\frac{2}{3}$ \\
\hline
\end{tabular}

Notice that

$$
\left\langle\chi^{\rho}\left|\boldsymbol{\sigma}_{3}\right| \chi^{\rho}\right\rangle-\left\langle\chi^{\lambda}\left|\boldsymbol{\sigma}_{3}\right| \chi^{\lambda}\right\rangle=-4\left\langle\chi^{\lambda}\left|\boldsymbol{\sigma}_{3}\right| \chi^{\lambda}\right\rangle
$$

The ratio can be written as

$$
\mathcal{R}=f_{\mathcal{R}} \frac{\sum_{m}\left\langle J, \frac{1}{2} \mid 1, m, \frac{3}{2}, \frac{1}{2}-m\right\rangle\left\langle\chi^{\lambda}\left|\boldsymbol{\sigma}_{3}\right| \chi^{S}\right\rangle \bullet\left\langle\psi^{S}\left|\boldsymbol{p}_{3} e^{i q . r_{3}}\right| \psi^{\lambda}\right\rangle}{\sum_{m}\left\langle J, \frac{1}{2} \mid 1, m, \frac{3}{2}, \frac{1}{2}-m\right\rangle\left\langle\chi^{\lambda}\left|\boldsymbol{\sigma}_{3}\right| \chi^{\lambda}\right\rangle \bullet\left\langle\psi^{S}\left|\boldsymbol{p}_{3} e^{i q . r_{3}}\right| \psi^{\lambda}\right\rangle} .
$$

Calculation of the matrix elements goes as follows.

$$
\begin{array}{r}
\sum_{m}\left\langle J, \frac{1}{2} \mid 1, m, \frac{3}{2}, \frac{1}{2}-m\right\rangle\left\langle\chi^{\lambda}\left|\boldsymbol{\sigma}_{3}\right| \chi^{S}\right\rangle \bullet\left\langle\psi^{S}\left|\boldsymbol{p}_{3} e^{i q \cdot r_{3}}\right| \psi^{\lambda}\right\rangle= \\
\left\langle J, \frac{1}{2} \mid 1,0, \frac{3}{2}, \frac{1}{2}\right\rangle\left\langle\chi_{\frac{1}{2}}^{\lambda}\left|\boldsymbol{\sigma}_{3}^{0}\right| \chi_{\frac{1}{2}}^{S}\right\rangle\left\langle\psi^{S}\left|\boldsymbol{p}_{3}^{0} e^{i q \cdot r_{3}}\right| \psi_{1,0}^{\lambda}\right\rangle+ \\
\left\langle J, \frac{1}{2} \mid 1,1, \frac{3}{2},-\frac{1}{2}\right\rangle\left\langle\chi_{\frac{1}{2}}^{\lambda}\left|\boldsymbol{\sigma}_{3}^{+}\right| \chi_{-\frac{1}{2}}^{S}\right\rangle\left\langle\psi^{S}\left|\boldsymbol{p}_{3}^{-} e^{i q \cdot r_{3}}\right| \psi_{1,1}^{\lambda}\right\rangle+ \\
\left\langle J, \frac{1}{2} \mid 1,-1, \frac{3}{2}, \frac{3}{2}\right\rangle\left\langle\chi_{\frac{1}{2}}^{\lambda}\left|\boldsymbol{\sigma}_{3}^{-}\right| \chi_{\frac{3}{2}}^{S}\right\rangle\left\langle\psi^{S}\left|\boldsymbol{p}_{3}^{+} e^{i q \cdot r_{3}}\right| \psi_{1,-1}^{\lambda}\right\rangle .
\end{array}
$$


For $\mathrm{J}=1 / 2$ :

$$
\begin{array}{r}
\sum_{m}\left\langle J, \frac{1}{2} \mid 1, m, \frac{3}{2}, \frac{1}{2}-m\right\rangle\left\langle\chi^{\lambda}\left|\boldsymbol{\sigma}_{3}\right| \chi^{S}\right\rangle \bullet\left\langle\psi^{S}\left|\boldsymbol{p}_{3} e^{i q \cdot r_{3}}\right| \psi^{\lambda}\right\rangle= \\
\frac{2 \sqrt{2}}{3 \sqrt{3}}\left\langle\psi^{S}\left|\boldsymbol{p}_{3}^{0} e^{i q \cdot r_{3}}\right| \psi_{1,0}^{\lambda}\right\rangle-\frac{1}{3 \sqrt{3}}\left\langle\psi^{S}\left|\boldsymbol{p}_{3}^{-} e^{i q \cdot r_{3}}\right| \psi_{1,1}^{\lambda}\right\rangle+\frac{1}{\sqrt{3}}\left\langle\psi^{S}\left|\boldsymbol{p}_{3}^{+} e^{i q \cdot r_{3}}\right| \psi_{1,-1}^{\lambda}\right\rangle= \\
\frac{2 \sqrt{2}}{3 \sqrt{3}}\left(\left\langle\psi^{S}\left|\boldsymbol{p}_{3}^{0} e^{i q \cdot r_{3}}\right| \psi_{1,0}^{\lambda}\right\rangle-\sqrt{2}\left\langle\psi^{S}\left|\boldsymbol{p}_{3}^{-} e^{i q \cdot r_{3}}\right| \psi_{1,1}^{\lambda}\right\rangle\right) .
\end{array}
$$

For $\mathrm{J}=3 / 2$ :

$$
\begin{array}{r}
\sum_{m}\left\langle\frac{3}{2}, \frac{1}{2} \mid 1, m, \frac{3}{2}, \frac{1}{2}-m\right\rangle\left\langle\chi^{\lambda}\left|\boldsymbol{\sigma}_{3}\right| \chi^{S}\right\rangle \bullet\left\langle\psi^{S}\left|\boldsymbol{p}_{3} e^{i q \cdot r_{3}}\right| \psi^{\lambda}\right\rangle= \\
\frac{2 \sqrt{2}}{3 \sqrt{15}}\left(\left\langle\psi^{S}\left|\boldsymbol{p}_{3}^{0} e^{i q \cdot r_{3}}\right| \psi_{1,0}^{\lambda}\right\rangle+\frac{1}{\sqrt{2}}\left\langle\psi^{S}\left|\boldsymbol{p}_{3}^{-} e^{i q \cdot r_{3}}\right| \psi_{1,1}^{\lambda}\right\rangle\right) .
\end{array}
$$

Notice that

$$
\left\langle\psi^{S}\left|\boldsymbol{p}_{3}^{-} e^{i q \cdot r_{3}}\right| \psi_{1,1}^{\lambda}\right\rangle=\left\langle\psi^{S}\left|\boldsymbol{p}_{3}^{+} e^{i q \cdot r_{3}}\right| \psi_{1,-1}^{\lambda}\right\rangle,
$$

where $p^{ \pm}=p^{x} \pm i p^{y}$.

Similarly,

$$
\begin{array}{r}
\sum_{m}\left\langle J, \frac{1}{2} \mid 1, m, \frac{1}{2}, \frac{1}{2}-m\right\rangle\left\langle\chi^{\lambda}\left|\boldsymbol{\sigma}_{3}\right| \chi^{\lambda}\right\rangle \bullet\left\langle\psi^{S}\left|\boldsymbol{p}_{3} e^{i q \cdot r_{3}}\right| \psi^{\lambda}\right\rangle= \\
\left\langle J, \frac{1}{2} \mid 1,0, \frac{1}{2}, \frac{1}{2}\right\rangle\left\langle\chi_{\frac{1}{2}}^{\lambda}\left|\boldsymbol{\sigma}_{3}^{0}\right| \chi_{\frac{1}{2}}^{\lambda}\right\rangle\left\langle\psi^{S}\left|\boldsymbol{p}_{3}^{0} e^{i q \cdot r_{3}}\right| \psi_{1,0}^{\lambda}\right\rangle+ \\
\left\langle J, \frac{1}{2} \mid 1,1, \frac{1}{2},-\frac{1}{2}\right\rangle\left\langle\chi_{\frac{1}{2}}^{\lambda}\left|\boldsymbol{\sigma}_{3}^{+}\right| \chi_{-\frac{1}{2}}^{S}\right\rangle\left\langle\psi^{S}\left|\boldsymbol{p}_{3}^{-} e^{i q \cdot r_{3}}\right| \psi_{1,1}^{\lambda}\right\rangle .
\end{array}
$$

For $\mathrm{J}=1 / 2$ :

$$
\begin{array}{r}
\sum_{m}\left\langle J, \frac{1}{2} \mid 1, m, \frac{1}{2}, \frac{1}{2}-m\right\rangle\left\langle\chi^{\lambda}\left|\boldsymbol{\sigma}_{3}\right| \chi^{\lambda}\right\rangle \bullet\left\langle\psi^{S}\left|\boldsymbol{p}_{3} e^{i q \cdot r_{3}}\right| \psi^{\lambda}\right\rangle= \\
\frac{1}{3 \sqrt{3}}\left(\left\langle\psi^{S}\left|\boldsymbol{p}_{3}^{0} e^{i q \cdot r_{3}}\right| \psi_{1,0}^{\lambda}\right\rangle-\sqrt{2}\left\langle\psi^{S}\left|\boldsymbol{p}_{3}^{-} e^{i q \cdot r_{3}}\right| \psi_{1,1}^{\lambda}\right\rangle\right) .
\end{array}
$$

For $\mathrm{J}=3 / 2$ :

$$
\begin{array}{r}
\sum_{m}\left\langle\frac{3}{2}, \frac{1}{2} \mid 1, m, \frac{1}{2}, \frac{1}{2}-m\right\rangle\left\langle\chi^{\lambda}\left|\boldsymbol{\sigma}_{3}\right| \chi^{\lambda}\right\rangle \bullet\left\langle\psi^{S}\left|\boldsymbol{p}_{3} e^{i q \cdot r_{3}}\right| \psi^{\lambda}\right\rangle= \\
-\frac{\sqrt{2}}{3 \sqrt{3}}\left(\left\langle\psi^{S}\left|\boldsymbol{p}_{3}^{0} e^{i q \cdot r_{3}}\right| \psi_{1,0}^{\lambda}\right\rangle+\frac{1}{\sqrt{2}}\left\langle\psi^{S}\left|\boldsymbol{p}_{3}^{-} e^{i q \cdot r_{3}}\right| \psi_{1,1}^{\lambda}\right\rangle\right) .
\end{array}
$$

Now, the constant $\mathcal{R}$ can be calculated for $\mathrm{J}=1 / 2$ :

$$
R_{S}=f_{\mathcal{R}} \frac{\frac{2 \sqrt{2}}{3 \sqrt{3}}}{\frac{1}{3 \sqrt{3}}}=2 \sqrt{2} f_{\mathcal{R}},
$$


and for $\mathrm{J}=3 / 2$ :

$$
R_{D}=-f_{\mathcal{R}} \frac{\frac{2 \sqrt{2}}{3 \sqrt{15}}}{\frac{\sqrt{2}}{3 \sqrt{3}}}=-\frac{2}{\sqrt{5}} f_{\mathcal{R}} .
$$

Below, we give the values of $f_{\mathcal{R}}$ for pseudoscalar mesons photoproduction processes, namely, $\gamma p \rightarrow \eta p, K^{+} \Lambda, K^{+} \Sigma^{\circ}, K^{\circ} \Sigma^{+}$:

$$
f_{\mathcal{R}}=\left\{\begin{array}{cc}
-\frac{1}{2 \sqrt{2}} & \text { for } \eta N \\
0 & \text { for } K \Lambda \\
-\sqrt{2} & \text { for } K \Sigma
\end{array}\right.
$$

Accordingly, the numerical values for the constant $\mathcal{R}$ are given in Table 4 Notice that the values of both $\mathcal{R}$ constants vanish for the $\gamma p \rightarrow K^{+} \Lambda$ channel, in agreement with the $\Lambda$ selection rule discussed in Ref. 35].

Table 4 Values of the $\mathcal{R}$ constant, within the Koniuk and Isgur [16] convention, for the $\eta$ and kaon photoproduction processes.

\begin{tabular}{cccc}
\hline & $\gamma p \rightarrow \eta p$ & $\gamma p \rightarrow K^{+} \Lambda$ & $\gamma p \rightarrow K^{+} \Sigma^{\circ}, K^{\circ} \Sigma^{+}$ \\
\hline $\mathcal{R}_{S}$ & -1 & 0 & -4 \\
$\mathcal{R}_{D}$ & $\frac{1}{\sqrt{10}}$ & 0 & $\frac{4}{\sqrt{10}}$ \\
\hline
\end{tabular}

Notice that in the present work, we have adopted the convention introduced by Koniuk and Isgur [16], where wave functions are in line with the SU(3) conventions of de Swart [0]. In this frame, e.g. for the process $\gamma p \rightarrow \eta p$, the constant $\mathcal{R}_{S}$ gets a negative value, and the relevant mixing angle for the $S$-wave, $\theta_{S}$, turns out positive. However, in line with the Hey et al. [42] analysis, Isgur and Karl in their early works [13,44,45, 46. used another convention, for which $\mathcal{R}_{S}=+1$ and $\theta_{S}<0$. In the literature both conventions are being used, often without explicit mention of the utilized convention.

Our final results relating the $S U(6) \otimes O(3)$ symmetry breaking coefficients and mixing angles are presented in Table 5 .

To end this section, we wish to emphasize that the photoproduction reactions in Table 5 are being extensively studied, since about one decade, both theoretically and experimentally. The chiral constituent quark model, briefly presented in Sec. 2.1 has been used to study $\gamma p \rightarrow \eta p$ [22,23,24,25,26] and $\gamma p \rightarrow K^{+} \Lambda$ [32] processes. The mixing angles, left as adjustable parameters, have been extracted [22,24] by fitting $\gamma p \rightarrow \eta p$ data 41,63, including polarization asymmetries. Those models embody all nucleon resonances given in Table 1 In next Section, we report on those results as well as on findings by various authors with respect to the mixing angles. 
Table 5 Relations among the symmetry breaking coefficients and mixing angles, Eqs. (18) to (21), for the $\eta$ and kaon photoproduction processes.

\begin{tabular}{cccc}
\hline & $\gamma p \rightarrow \eta p$ & $\gamma p \rightarrow K^{+} \Lambda$ & $\gamma p \rightarrow K^{+} \Sigma^{\circ}, K^{\circ} \Sigma^{+}$ \\
\hline$C_{S_{11}(1535)}$ & $\cos \theta_{S}\left(\cos \theta_{S}+\sin \theta_{S}\right)$ & $\cos ^{2} \theta_{S}$ & $\cos \theta_{S}\left(\cos \theta_{S}+4 \sin \theta_{S}\right)$ \\
$C_{S_{11}(1650)}$ & $\sin \theta_{S}\left(-\cos \theta_{S}+\sin \theta_{S}\right)$ & $\sin ^{2} \theta_{S}$ & $\sin \theta_{S}\left(-4 \cos \theta_{S}+\sin \theta_{S}\right)$ \\
$C_{D_{13}(1520)}$ & $\cos \theta_{D}\left(\cos \theta_{D^{-}} \frac{1}{\sqrt{10}} \sin \theta_{D}\right)$ & $\cos ^{2} \theta_{D}$ & $\cos \theta_{D}\left(\cos \theta_{D^{-}} \frac{4}{\sqrt{10}} \sin \theta_{D}\right)$ \\
$C_{D_{13}(1700)}$ & $\sin \theta_{D}\left(\frac{1}{\sqrt{10}} \cos \theta_{D}+\sin \theta_{D}\right)$ & $\sin ^{2} \theta_{D}$ & $\sin \theta_{D}\left(\sin \theta_{D^{+}}+\frac{4}{\sqrt{10}} \cos \theta_{D}\right)$ \\
\hline
\end{tabular}

\section{Determination of mixing angles}

In mid-seventies, Hey et al. 42. performed a comprehensive analysis of decay rates of baryon resonances belonging to the $\left[70,1^{-}\right]$and $\left[56,2^{+}\right]$representations of $S U(6) \otimes$ $O(3)$ into the ground state $\left[56,0^{+}\right]$baryons and the pseudoscalar mesons.

Based on the pioneer work by De Rujula et al. [43] a non-relativistic constituent quark approach was developed by Isgur, Karl, and collaborators [13, 14, 15, 16, 44, 45, 46. Isgur and Karl, using a harmonic oscillator confining potential with the OGE interaction found excellent [13,45] agreement with the extracted values from experimental decay rates. In the Isgur-Karl et al. approach, a major assumption is that the quark dynamics is subject to the gluon field, which provides a confining potential. However, within chiral perturbation theory, at low energy the effective degrees of freedom are mesons, instead of gluons.

Extensive investigations have been performed 47] considering the exchange of a pseudoscalar octet between light quarks generating the hyperfine structure. That approach has been generalized by Glozman, Plessas, Varga, and Wagenbrunn [4, 49] embodying the exchange of a nonent of vector mesons and a scalar meson. Within that scope, Glozman and Riska [7], generalizing one-pion-exchange (OPE) mechanism, attributed the spin-dependent coupling between constituent quarks to Goldstone-bosonexchange (GBE).

In addition to the above approaches, there are other versions of CQM, according to the embodied quark dynamics, such as algebric [50], hypercentral [20,51, and instanton [52. This latter, a powerful formalism of relativistically covariant constituent quark model, has been extensively developed by the Bonn group 52. That approach is based on the three-fermion Bethe-Salpeter equation, where the confinement is generated by an instantaneous string-like three-body potential. The results of those works allow the authors to account for the spectrum of known resonances, predict missing ones, and put forward an explanation for those not yet observed states, due to their vanishing couplings to the $\pi N$ or $\bar{K} N$ systems, as suggested in Refs. 16,53].

Another covariant model [54] based on the Dyson-Schwinger equations, relates the confinement to the analytical properties of QCD's Schwinger functions, and offers a reliable frame to interpret baryon data directly in terms of current quarks and gluons. Moreover, this approach establishes a link between the phenomenology of dressed current quarks and Lattice QCD [54,55]. 
Table 6 Mixing angles for the two $S_{11}$ and the two $D_{13}$ states around 1.5 to $1.7 \mathrm{GeV}$.

\begin{tabular}{lccl}
\hline Approach & $\Theta_{S}(\mathrm{deg})$ & $\Theta_{D}(\mathrm{deg})$ & Authors (Ref.) \\
\hline Decay rates analysis & -31.9 & +10.4 & Hey et al. [42] \\
OGE (H.O.) & -32 & +6 & Isgur-Karl [13] 45]; Chizma-Karl [57] \\
OGE (B.M.) & -32 & +4 & Chizma [58] \\
OPE (H.O.) & \pm 13 & \pm 8 & Glozman-Riska [77; Isgur [56] \\
OPE (H.O.) & +26 & -53 & Chizma-Karl [57] \\
OPE (B.M.) & +29 & -47 & Chizma [58] \\
RCQM & $+38 \pm 4$ & $+10 \div 15$ & Capstick-Roberts [59] \\
$1 / N_{c}$ expansion & +22 & +28 & Pirjol-Schat [60] \\
CQM $(\gamma p \rightarrow \eta p)$ & -27 & +5 & Saghai-Li [22] \\
& -35 & +15 & He et al. 24]
\end{tabular}

In Table 6, results reported in the literature for the mixing angles are summarized and compared with their experimental values 42 (first row). The rows two to six embody results from OGE 13, 45,57 and OPE / GBE 47, 56,57 approaches built on harmonic-oscillator ( $\mathrm{HO}$ ) basis for the orbital wave functions or the Bag model (BM) [58. Results obtained [59] within a relativized constituent quark model (RCQM), based on $\mathrm{HO}$ and OGE, give (row seven) comparable values for mixing angles as the non-relativistic CQM, albeit within a sign difference for the $S_{11}$ resonances due to the use of Koniuk and Isgur [16] convention. Notice that the other results coming from CQM/OGE approaches use the conventions introduced by Hey et al. 42.

The $1 / N_{c}$ expansion approach has also been extensively applied to the determination of mixing angles 60,61, 62. The outcomes are within the following ranges: $0^{\circ} \leq \Theta_{S} \leq 35^{\circ}$ and $0^{\circ} \leq \Theta_{D} \leq 45^{\circ}$, with typical values given in row eight.

In the last two rows of Table 6 we report the results from our phenemenological chiral constituent quark model investigation the $\gamma p \rightarrow \eta p$ process, where the $S U(6) \otimes$ $O(3)$ symmetry breaking coefficients are left as adjustable parameters. A first study 22 used the data base [63] available in 2000, limited to total center-of-mass energies $W \leq$ $2 \mathrm{GeV}$, and led to $\Theta_{S}=-27^{\circ}$ and $\Theta_{D}=+5^{\circ}$. Since then, much copious and accurate experimental results 41 have been released up to $W \approx 2.6 \mathrm{GeV}$. That data base has been investigated within a more advanced approach 24, embodying all known resonances. The extracted values are $\Theta_{S}=-35^{\circ}$ and $\Theta_{D}=+15^{\circ}$, and turn out to be in good agreement with experimental values and those calculated by Isgur and Kar1. However, more investigation are needed for kaon photoproduction processes, where contributions from the second $S_{11}$ resonance are more significant (Table 5) than in $\gamma p \rightarrow \eta p$. A systematic study in both $\eta$ and kaon photoproduction reactions will provide more accurate information on the mixing angles.

\footnotetext{
1 Notice that within Isgur-Karl convention, those angles lead to the following values for the configuration mixing coefficients (Eqs. (18) to (21), with $\mathcal{R}_{S}=1$ ): $C_{S_{11}(1535)}=1.14, C_{S_{11}(1650)}$ $=-0.14, C_{D_{13}(1520)}=0.85$, and $C_{D_{13}(1700)}=0.15$. Given that the unbroken $S U(6) \otimes O(3)$ symmetry predicts for those coefficients $\left|C_{S_{11}(1535)}\right|=\left|C_{D_{13}(1520)}\right|=1$, and $C_{S_{11}(1650)}=$ $C_{D_{13}(1700)}=0$, then the symmetry breaking effects come out to be around $15 \%$.
} 


\section{Conclusions}

In this paper, within a chiral constituent quark approach, we reported on the derivation of relations between the transition amplitudes in the $\eta$ and kaon photoproduction channels and the two most widely investigated mixing angles $\left(\Theta_{S}\right.$ and $\left.\Theta_{D}\right)$ for the resonances $S_{11}(1535)$ and $S_{11}(1650) ; D_{13}(1520)$ and $D_{13}(1700)$. The extracted mixing angles from the photoproduction process $\gamma p \rightarrow \eta p$ are in good agreement with those obtained from the resonances decay data 42 .

The mixing angles from the $S$ - and $D$-wave resonance offer important tools to test various quark models, which may provide us with insights into the underlying dynamics of the quarks interations. This program requires systematic studies both on baryon spectroscopy and on the properties of strong, weak and electromagnetic transitions. A recent work 62 puts forward a quark Hamiltonian embodying a mix of the OGE and GBE interactions. Another important development in this realm comes from recent investigations concluding that the SU(6) symmetry breaking effects can be attributed, partly to spin- and flavor-dependent interactions between the quarks, and partly to loop effects, emphasizing the need for careful treatment of mixing mechanisms 64.

Results from other approaches were briefly discussed. We found that the extracted mixing angle from the $\eta$ photoproduction are consistent with the results from the IsgurKarl [13,45] model. More investigations are needed in the kaon photoproduction, where contributions from the second $S_{11}$ state are significant.

Acknowledgements We wish to thank Qiang Zhao for fruitful exchanges.

\section{References}

1. H. L. Anderson, E. Fermi, E. A. Long and D. E. Nagle, Phys. Rev. 85, 936 (1952).

2. K. A. Brueckner, Phys. Rev. 86, 106 (1952).

3. H. L. Anderson, E. Fermi, R. Martin and D. E. Nagle, Phys. Rev. 91, 155 (1953).

4. H. L. Anderson, W. C. Davidon and U. E. Kruse, Phys. Rev. 100, 339 (1955).

5. J. Ashkin, J. P. Blaser, F. Feiner, and M. O. Stern, Phys. Rev. 101, 1149 (1956).

6. M. Gell-Mann and Y. Neemam, The eightfold way: a review with a collection of reprints, W. A. Benjamin Publisher (1964).

7. G. Zweig, CERN preprint 8409/Th. 412, unpublished.

8. F. Gürsey, A. Pais, and L. A. Radicati, Phys. Rev. Lett. 13, 173 (1964); A. Pais, Phys. Rev. Lett. 13, 175 (1964); F. Gürsey and L. A. Radicati, Phys. Rev. Lett. 13, 299 (1964); T. K. Kuo and Tsu Yao, Phys. Rev. Lett. 13, 415 (1964); Mirza A. Baqi Bég and Virendra Singh, Phys. Rev. Lett. 13, 418 (1964); B. Sakita, Phys. Rev. 136, B1756 (1964).

9. K. T. Mahanthappa and G. Sudarshan, Phys. Rev. Lett. 14, 163 (1965).

10. L. A. Copley, G. Karl and E. Obryk, Phys. Rev. D 4, 2844 (1971).

11. R. P. Feynman, M. Kislinger and F. Ravndal, Phys. Rev. D 3, 2706 (1971).

12. C. Amsler et al. [Particle Data Group], Phys. Lett. B 667, 1 (2008).

13. N. Isgur and G. Karl, Phys. Lett. B 72, 109 (1977).

14. N. Isgur, G. Karl and R. Koniuk, Phys. Rev. Lett. 41, 1269 (1978); Erratum-ibid. 45, $1738(1980)$.

15. N. Isgur and G. Karl, Phys. Rev. D 20, 1191 (1979).

16. R. Koniuk and N. Isgur, Phys. Rev. D 21, 1868 (1980); Erratum-ibid. D 23, 818 (1981).

17. S. Capstick and W. Roberts, Phys. Rev. D 49, 4570 (1994).

18. R. Bijker, F. Iachello and A. Leviatan, Annals Phys. 236, 69 (1994).

19. S. Capstick and W. Roberts, Prog. Part. Nucl. Phys. 45, S241 (2000).

20. M. M. Giannini, E. Santopinto and A. Vassallo, Eur. Phys. J. A 12, 447 (2001).

21. T. Melde, W. Plessas and B. Sengl, Phys. Rev. D 77, 114002 (2008).

22. B. Saghai and Z. p. Li, Eur. Phys. J. A 11, 217 (2001). 
23. Q. Zhao, B. Saghai and Z. p. Li, J. Phys. G 28, 1293 (2002).

24. J. He, B. Saghai, Z. Li, Q. Zhao and J. Durand, Eur. Phys. J. A 35, 321 (2008).

25. J. He, B. Saghai and Z. Li, Phys. Rev. C 78, 035204 (2008).

26. Jun He and B. Saghai, Phys. Rev. C 80, 015207 (2009).

27. W. T. Chiang, S. N. Yang, L. Tiator, M. Vanderhaeghen and D. Drechsel, Phys. Rev. C 68, 045202 (2003).

28. R. Arndt, W. Briscoe, I. Strakovsky and R. Workman, Eur. Phys. J. A 35, 311 (2008).

29. A. V. Anisovich, A. Sarantsev, O. Bartholomy, E. Klempt, V. A. Nikonov and U. Thoma, Eur. Phys. J. A 25, 427 (2005).

30. T. Feuster and U. Mosel, Phys. Rev. C 59, 460 (1999); V. Shklyar, H. Lenske and U. Mosel, Phys. Lett. B 650, 172 (2007).

31. R. Shyam and O. Scholten, arXiv:0808.0632 [nucl-th].

32. B. Julia-Diaz, B. Saghai, F. Tabakin, W. T. Chiang, T. S. Lee and Z. Li, Nucl. Phys. A 755, 463 (2005); B. Julia-Diaz, B. Saghai, T. S. Lee and F. Tabakin, Phys. Rev. C 73, 055204 (2006); B. Saghai, J. C. David, B. Julia-Diaz and T. S. Lee, Eur. Phys. J. A 31 , $512(2007)$.

33. A. V. Sarantsev, V. A. Nikonov, A. V. Anisovich, E. Klempt and U. Thoma, Eur. Phys. J. A 25, 441 (2005).

34. V. Shklyar, H. Lenske and U. Mosel, Phys. Rev. C 72, 015210 (2005).

35. Q. Zhao and F. E. Close, Phys. Rev. D 74, 094014 (2006).

36. Z. p. Li, H. x. Ye and M. h. Lu, Phys. Rev. C 56, 1099 (1997).

37. A. Manohar and H. Georgi, Nucl. Phys. B 234, 189 (1984).

38. Z. p. Li and B. Saghai, Nucl. Phys. A 644, 345 (1998).

39. R. G. Moorhouse, Phys. Rev. Lett. 16, 772 (1966).

40. J. J. de Swart, Rev. Mod.Phys. 35, 916 (1963).

41. M. Dugger et al. [CLAS Collaboration], Phys. Rev. Lett. 89, 222002 (2002); V. Crede et al. [CB-ELSA Collaboration], Phys. Rev. Lett. 94, 012004 (2005); T. Nakabayashi et al., Phys. Rev. C 74, 035202 (2006); O. Bartalini et al. [The GRAAL collaboration], Eur. Phys. J. A 33, 169 (2007); O. Bartholomy et al. [CB-ELSA Collaboration], Eur. Phys. J. A 33, 133 (2007); D. Elsner et al. [CBELSA Collaboration and TAPS Collaboration], Eur. Phys. J. A 33, 147 (2007)

42. A. J. G. Hey, P. J. Litchfield and R. J. Cashmore, Nucl. Phys. B 95, 516 (1975).

43. A. De Rujula, H. Georgi and S. L. Glashow, Phys. Rev. D 12, 147 (1975).

44. N. Isgur and G. Karl, Phys. Lett. B 74, 353 (1978).

45. N. Isgur and G. Karl, Phys. Rev. D 18, 4187 (1978).

46. N. Isgur and G. Karl, Phys. Rev. D 19, 2653 (1979); Erratum-ibid. D 23, 817 (1981).

47. L. Y. Glozman and D. O. Riska, Phys. Rept. 268, 263 (1996).

48. L. Y. Glozman, W. Plessas, K. Varga and R. F. Wagenbrunn, Phys. Rev. D 58, 094030 (1998).

49. L. Y. Glozman, Nucl. Phys. A 663, 103 (2000).

50. R. Bijker, F. Iachello and A. Leviatan, Annals Phys. 236, 69 (1994); R. Bijker, F. Iachello and A. Leviatan, Phys. Rev. D 55, 2862 (1997).

51. M. M. Giannini, E. Santopinto and A. Vassallo, Prog. Part. Nucl. Phys. 50, 263 (2003); M. Gorchtein, D. Drechsel, M. M. Giannini, E. Santopinto and L. Tiator, Phys. Rev. C 70, 055202 (2004).

52. U. Loring, K. Kretzschmar, B. C. Metsch and H. R. Petry, Eur. Phys. J. A 10, 309 (2001); U. Loring, B. C. Metsch and H. R. Petry, Eur. Phys. J. A 10, 395 (2001); U. Loring, B. C. Metsch and H. R. Petry, Eur. Phys. J. A 10, 447 (2001); B. Metsch, Eur. Phys. J. A 35, 275 (2008)

53. S. Capstick and W. Roberts, Phys. Rev. D 47, 1994 (1993).

54. C. D. Roberts, Prog. Part. Nucl. Phys. 61, 50 (2008).

55. see e.g. C. Alexandrou, arXiv:0906.4137 [hep-lat], and references therein.

56. N. Isgur, Phys. Rev. D 62, 054026 (2000)

57. J. Chizma and G. Karl, Phys. Rev. D 68, 054007 (2003).

58. J. Chizma, Ph.D Thesis, The University of Guelph (2004), unpublished.

59. S. Capstick and W. Roberts, Fizika B 13, 271 (2004).

60. D. Pirjol and C. Schat, Phys. Rev. D 67, 096009 (2003).

61. N. N. Scoccola, J. L. Goity and N. Matagne, Phys. Lett. B 663, 222 (2008); D. Pirjol and C. Schat, Phys. Rev. D 78, 034026 (2008); and references therein.

62. D. Pirjol and C. Schat, Phys. Rev. Lett. 102, 152002 (2009). 
63. S. A. Dytman et al., Phys. Rev. C 51, 2710 (1995); J. W. Price et al., Phys. Rev. C 51, R2283 (1995); B. Krusche et al., Phys. Rev. Lett. 74, 3736 (1995); A. Bock et al., Phys. Rev. Lett. 81, 534 (1998); J. Ajaka et al., Phys. Rev. Lett. 81, 1797 (1998); F. Renard et al., Phys. Lett. B 528, 215 (2002).

64. S. Capstick et al., Eur. Phys. J. A 35, 253 (2008). 